\title{
Hepatic Echinococcal Cysts: A Review
}

\author{
Tina Pakala ${ }^{1}$, Marco Molina ${ }^{2}$ and George Y. Wu*1 \\ ${ }^{1}$ Department of Medicine, Division of Gastroenterology-Hepatology, University of Connecticut Health Center, Farmington, CT, \\ USA; ${ }^{2}$ Department of Radiology, University of Connecticut Health Center, Farmington, CT, USA
}

\begin{abstract}
Cystic echinococcosis (CE) is a widely endemic helminthic disease caused by infection with metacestodes (larval stage) of the Echinococcus granulosus tapeworm. E. granulosus are common parasites in certain parts of the world, and are present on every continent with the exception of Antarctica. As a result, a large number of people are affected by CE. The increased emigration of populations from endemic areas where prevalence rates are as high as $5-10 \%$ and the relatively quiescent clinical course of CE pose challenges for accurate and timely diagnoses. Upon infection with $\mathrm{CE}$, cyst formation mainly occurs in the liver (70\%). Diagnosis involves serum serologic testing for antibodies against hydatid antigens, but preferably with imaging by ultrasound or CT/ MRI. Treatment methods include chemotherapy with benzimidazole carbamates and/or surgical approaches, including percutaneous aspiration injection and reaspiration. The success of these methods is influenced by the stage and location of hepatic cysts. However, CE can be clinically silent, and has a high risk for recurrence. It is important to consider the echinococcal parasite in the differential diagnosis of liver cystic lesions, especially in patients of foreign origin, and to perform appropriate long-term follow-ups. The aim of this review is to highlight the epidemiology, natural history, diagnostic methods, and treatment of liver disease caused by E. granulosus. (C) 2016 The Second Affiliated Hospital of Chongqing Medical University. Published by XIA \& HE Publishing Inc. All rights reserved.
\end{abstract}

\section{Host/parasite life cycle}

Echinococcus granulosus is a small tapeworm that typically infects carnivores, such as dogs, foxes, and wolves, after the consumption of offal from infected intermediate hosts, such as sheep or pigs. Upon entering the small intestine, the parasite remains firmly attached to the mucosa, and later sheds gravid proglottids that are excreted in the infected animal's feces. ${ }^{1}$ Within each proglottid, there are hundreds of eggs. These eggs can then be ingested by intermediate hosts

Keywords: Anaphylaxis; Echinococcus; Hydatid cyst; Tapeworm.

Abbreviations: CE, cystic echinococcosis; Ig, immunoglobulin; IWGE, Informal Working Group Classification on Echinococcus; PAIR, percutaneous aspiration injection and reaspiration; WHO, World Health Organization.

Received: 21 October 2015; Revised: 15 December 2015; Accepted: 16 December 2015

DOI: $10.14218 / \mathrm{JCTH} .2015 .00036$.

* Correspondence to: George Y. Wu, Department of Medicine, Division of Gastroenterology-Hepatology, University of Connecticut Health Center, 263 Farmington Ave, Farmington, CT 06030, USA. Tel: +1-860-679-2509, Fax: +1-860679-8582, E-mail: wu@nso.uchc.edu where they mature into cysts and daughter cysts, such as in sheep that acquire the infection by grazing upon grass contaminated with dog feces containing the eggs. Human infection does not occur by the handling or ingestion of meat or viscera from infected sheep. Rather, humans are accidental intermediate hosts that become infected either by direct contact with a dog contaminated with egg-bearing feces or by ingesting water, food, or soil contaminated with such feces.

In human infection, the first stage is the asymptomatic incubation period, during which ingested eggs release oncospheres that are able to penetrate the human intestinal wall. These oncospheres enter the portal venous system, which provides access to the liver, lungs, and various other organs. ${ }^{3,4,10}$ Next, the oncospheres begin cyst development. ${ }^{2,3}$ Cysts are usually unilocular, and can range anywhere from $1 \mathrm{~cm}$ to $15 \mathrm{~cm}$ in diameter. In hepatic cystic echinococcosis (CE), cyst growth ranges from $1-2 \mathrm{~mm}$ to $10 \mathrm{~mm}$ per year. They also tend to affect the right lobe more frequently than the left lobe due to the nature of portal blood flow. The cysts are composed of two derived layers of membrane: an inner, nucleated, germinal membrane, and an outer, acellular, laminated layer. The immune system responds to the cyst by forming a calcified fibrous capsule around it, which is the layer that is most often visualized on imaging studies. ${ }^{4}$ The cyst enlarges to form a combination of protoscolices (future heads of the adult worms) and daughter cysts. The combination of many protoscolices and cystic fluid appears grain-like on ultrasound imaging, and is thus termed "hydatid sand." Animals that consume organs infected with protoscolices will become definitive hosts, as the protoscolices attach firmly to the host's intestine, and then develop into an adult worm with a scolex (head), neck, and proglottids. ${ }^{2,4,42}$

With $E$. granulosus infection, cysts most often occur in the liver $(70 \%)$ or lungs $(20 \%)$. However, $10 \%$ of cysts can be found anywhere in the body, including the spleen (6\%), heart $(2 \%)$, kidney $(2 \%)$, and brain $(<2 \%)$. E. granulosus infections usually present as solitary cysts, and have single-organ involvement. In $10-15 \%$ of patients, there can be involvement of two organs depending on the specific geographic region and strain of parasite. ${ }^{4}$

\section{Epidemiology of CE}

According to the World Health Organization (WHO), E. granulosus is endemic in areas of South America, Eastern Europe, Russia, the Middle East, and China, where human incidence rates are as high as 50 per 100,000 person-years. In certain areas, such as slaughterhouses in South America, prevalence varies from $20 \%$ to as high as $95 \% .{ }^{19}$ The type of strains available and the typical intermediate host vary by region. The most common intermediate hosts are farm 
animals, such as sheep, goats, swine, camels, horses, and cattle, as well as mule deer. Of these, small ruminants, such as sheep and goats, are the most commonly affected. ${ }^{46}$ The sheep strain (G1) is the one most frequently associated with human echinococcal cysts. ${ }^{4}$

The incidence of surgical cases of echinococcosis reflects only a fraction of the number of infected hosts, which, in turn, is only a fraction of the actual prevalence in endemic areas. Echinococcosis particularly impacts the human population in developing countries, where treatments are not always physically or financially feasible. CE also takes a significant toll on global livestock production, with losses of as much as two billion dollars annually. ${ }^{19,43}$

\section{Symptoms of CE}

CE can go undetected for many years due to the slow growth and development of cysts and the response of the host's immune system. ${ }^{5,6}$ Depending on the size and location, cysts can eventually exert pressure on nearby structures, producing abdominal discomfort and pain. ${ }^{1,4,10}$ For example, cysts in the liver can compress bile ducts, causing obstruction that can manifest as obstructive jaundice, abdominal pain, anorexia, and pruritus. ${ }^{17}$ When in the lungs, cysts can irritate the membranes leading to chronic cough, dyspnea, pleuritic chest pain, and hemoptysis. ${ }^{4,18}$

Cyst rupture or leakage can cause immunologic symptoms from the initiation of an immunoglobulin ( $\mathrm{Ig}$ )E response, leading to allergic reactions most frequently characterized by hives, flushing, and mucous membrane swelling. ${ }^{1} \mathrm{~A}$ major rupture can cause a life-threatening anaphylactic reaction. ${ }^{17}$ Ruptured cysts can release viable cystic contents and protoscolices into the peritoneum, resulting in secondary hyatidosis. ${ }^{18}$ Thus, infectious symptoms can manifest as sepsis, either due to the primary infection or to a secondary infection from leakage into the biliary tree. In one study, bacterial superinfection was found in $7.3 \%(37 / 503)$ of patients diagnosed with $\mathrm{CE}^{9}{ }^{9}$ Four of these patients developed severe sepsis, and two patients died. Bacteria most commonly seen in the liver cyst infections included Escherichia coli, Enterococcus, and Streptococcus viridans.

\section{Diagnosis of CE}

Diagnosis of CE is achieved by a combination of serologic tests and imaging, usually in conjunction with a history of exposure or immigration from an endemic area. ${ }^{18}$ Diagnosis from simple serum studies is difficult because of the low sensitivity of the tests, which is frequently due to undetectable immune responses. ${ }^{6,7}$ Immune responses depend on the location, cyst wall intactness, and viability of the organisms. Serum liver enzyme tests also have low sensitivities, and are frequently unreliable in determining the underlying severity of the infection. Moreover, serum liver enzyme tests are abnormal in only $40 \%$ of CE infected patients. When present, alkaline phosphatase is commonly elevated, while aspartate/alanine transaminase ratios and bilirubin levels typically remain within the normal limits. Complete blood count tests may be helpful, as eosinophilia is found in these patients. ${ }^{18}$

\section{Serum assays}

In humans, infection with Echinococcus induces an antibody response, most commonly IgG (predominantly IgG1 and
IgG4), followed by IgM, IgA, and IgE. However, in approximately $30-40 \%$ of patients, no antibodies of any kind are detectable, even in individuals who have circulating parasitic antigens. ${ }^{6}$ These data suggest that the infection may be associated with an inhibition of the host immune response, possibly at the B cell level or by a T cell-mediated mechanism. It is also possible that the impermeability of the cyst wall to the host defense system plays a role in allowing the parasite to evade immune detection and response. ${ }^{10}$ Furthermore, the cyst may allow the organism to actively suppress the host's immune system. ${ }^{6}$

A number of detection assays for IgG, IgM, and IgE antibodies to hydatid antigens have been described. The currently available antibody detection assays include immunoelectrophoresis, ELISA, and immunoblots, which utilize native and recombinant antibodies and a hydatid fluid fraction. Immunoblots reportedly have the highest sensitivity $(80 \%)$, followed by ELISA ( $72 \%$ ) and immunoelectrophoresis $(31 \%) .{ }^{16}$ One study that examined 151 hepatic CE patients found that ELISA and indirect hemagglutination results were influenced by the number and size of cysts, cyst activity, and treatment $\leq 12$ months before serum collection. ${ }^{42}$ If the preliminary test with IgG ELISA is negative and there are no imaging or other signs of $C E$, patients do not require further work up. However, imaging results suggestive of CE in a seronegative patient necessitate repeated and extended serologic testing or consideration of cyst puncture, as well as consideration for medical and/or surgical intervention if the patient is symptomatic. ${ }^{2,35,37}$ In patients who are seropositive and have positive imaging findings, a secondary antibody test is performed, using either an Arc 5 test, IgG4-ELISA, or immunoblot for antibodies against $E$. granulosus antigens. Secondary tests are used to rule out false-positive cross-reactivities. ${ }^{8}$

Although antibody detection assays tend to have higher sensitivities (up to $97 \%$ ) when compared to antigen assays, ${ }^{15}$ they do not distinguish between active and past infections. Therefore, assays for antigens are preferred, as they are not only more specific, but levels have been shown to reflect improvement in surgically treated patients. ${ }^{13} \mathrm{~A}$ disadvantage of these assays is the variability in sensitivity rates, which range from $33 \%$ to $85 \% .{ }^{12,13}$ This variability may be due to the structure of calcified cysts, concealment of cysts by surrounding normal tissue, or the fact that antigen-antibody complexes are not easily detected by assays. ${ }^{14}$

In actuality, many of the serologic tests that have been developed are applicable only for research purposes, and are not broadly employable in clinical settings, especially in developing countries. Therefore, there is an emphasis on imaging modalities for diagnosing CE.

\section{Imaging}

The findings on imaging vary depending on the stage of the cyst. As several classification schemes exist for staging $\mathrm{CE}_{\text {, }}$ the WHO developed a standardized classification system. ${ }^{19}$ This system, originally developed by Gharbi and colleagues in 1981, was revised by the WHO-Informal Working Group Classification on Echinococcus (IWGE) and is currently the screening method of choice. Table 1 lists the classifying features for the development of CE. ${ }^{21}$ Such a classification system enables a standardized approach to treatment based on diagnosed stage. 
Pakala T. et al: Hepatic echinococcal cysts

Table 1. Ultrasound classification

\begin{tabular}{|c|c|c|c|}
\hline \multicolumn{2}{|c|}{ Classification type } & \multirow[b]{2}{*}{ Classifying features } & \multirow[b]{2}{*}{ Stage } \\
\hline Gharbi & WHO-IWGE & & \\
\hline I & CE1 & Univesicular fluid collection/simple cyst & Active \\
\hline III & CE2 & $\begin{array}{l}\text { Multivesicular fluid collection with multiple } \\
\text { daughter cysts or septae (honeycomb) }\end{array}$ & Active \\
\hline II & CE3 A & $\begin{array}{l}\text { Fluid collection with membrane detached } \\
\text { (water lily sign) }\end{array}$ & Transitional \\
\hline III & CE3 B & Daughter cysts in solid matrix & Transitional \\
\hline IV & CE4 & $\begin{array}{l}\text { Cysts with heterogeneous matrix, } \\
\text { no daughter cysts }\end{array}$ & Inactive/degenerative \\
\hline V & CE5 & Solid cystic wall & Inactive/degenerative \\
\hline
\end{tabular}

WHO-IWGE, World Health Organization-Informal Working Group Classification on Echinococcus.

Radiology

Calcification can be seen on radiographs in up to $30 \%$ of $\mathrm{CE}$ cases. ${ }^{38}$ The calcifications are usually curvilinear or ring-like, and are deposited in the pericyst. Calcification can progress throughout all stages of CE. However, it is implied that once a cyst is entirely calcified, the pathogen is inactive/dead. ${ }^{2,38}$

\section{Ultrasound}

Ultrasound has become a widely used modality for CE detection. Diagnostic accuracies for ultrasound reach close to $90 \%$, depending on user technique and experience. ${ }^{20}$ This is currently the screening method of choice, due in part to accessibility even in small, rural medical centers, cost containments, and portability of the device. Ultrasound is not only helpful for diagnosis, but in post-treatment monitoring.

On ultrasound, the cyst wall usually has a hypoechoic layer, flanked by an echogenic line on either side. ${ }^{38} \mathrm{~A}$ simple, unilocular cyst may not demonstrate an internal structure. However, multiple punctate echogenic foci are often present within the $\mathrm{CE}$, but are only visualized upon repositioning of the patient. These foci represent hydatid sand, a combination of fluid and protoscolices, which have recently ruptured from a vesicle within the cyst. ${ }^{2,18}$ An endocyst can also detach from the pericyst. This may appear as a well-defined cystic lesion with a localized split in the wall and "floating membranes" within the cystic cavity; complete detachment observed by ultrasonography is referred to as the water lily sign.2,38 Multivesicular cysts are fluid collections that often appear in a honeycomb pattern with multiple septa. The septa represent the walls of the daughter cysts, which appear as cysts within a cyst. ${ }^{2}$ Daughter cysts separated by the hydatid matrix can produce a "wheel-spoke pattern." The matrix is composed of detached membranes, which may appear as serpentine linear structures, broken daughter vesicles, scolices, and hydatid sand. $2,35,38$ Ultrasound has the highest sensitivity for the detection of membranes, septa, and hydatid sand within the CE. ${ }^{2}$

Once the matrix fills the cyst, the cyst can appear as a solid mass. In order to distinguish a cyst filled by matrix, daughter vesicles or membranes should be sought within the lesion. ${ }^{2}$ Calcification of cysts typically occurs within the wall, and appears hyperechoic with posterior acoustic shadowing. Severe calcification restricts ultrasound penetration, thus, only the anterior portion of the CE can then be visualized. ${ }^{2}$
CT

Although ultrasound is an excellent tool for initial diagnosis, sonographic failures can occur for a multitude of reasons, including obesity, excessive bowel gas, and previous surgeries. CT has a sensitivity rate approaching $94 \%,{ }^{22}$ and plays a crucial role during the perioperative period for detection of complications, such as biliary and vascular involvement, cyst ruptures, and underlying infection. ${ }^{17,23}$ In addition, CT can reveal many of the same findings that can be seen by ultrasonography.

CE fluid appears as water in attenuation (approximately 0 Hounsfield Units) (Fig. 1). Calcification in the cyst wall or internal septa can appear hyperdense on non-contrast CT (Fig. 2). Faint calcification may be obscured by intravenous iodinated contrast. Detached laminated membranes from the pericyst can be seen as linear, hyperdense areas within the CE
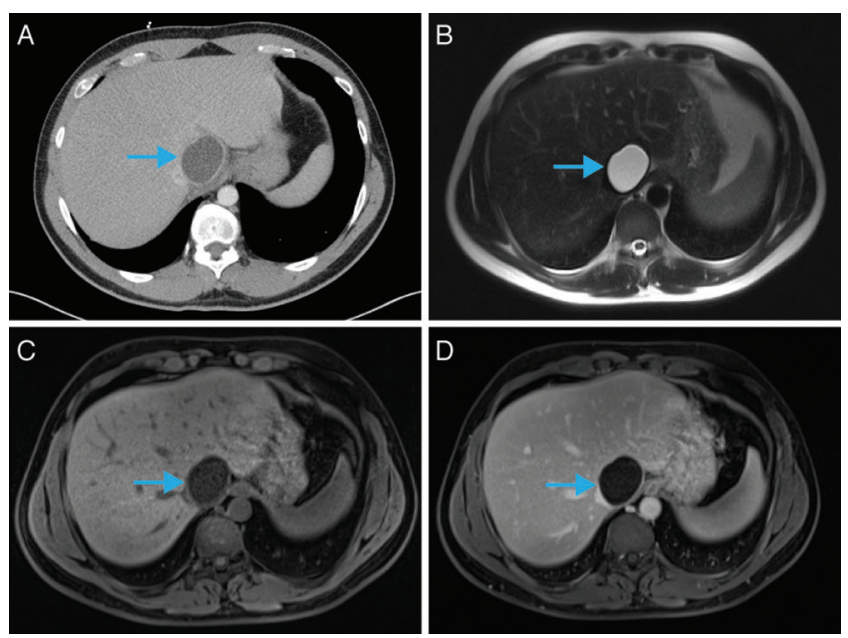

Fig. 1. Unilocular hepatic hydatid cyst (blue arrow) on CT and MRI. A: Contrast-enhanced CT: cystic lesion is hypodense with a thickened, enhancing rim; B: T2-weighted MRI: cystic mass is hyperintense with characteristic lowsignal intensity rim, probably representing the collagen-rich, outer layer (pericyst) of the hydatid cyst; C. Noncontrast T1-weighted MRI with fat saturation: cystic lesion is hypointense; D: Postcontrast T1-weighted MRI with fat saturation: cystic lesion demonstrates an enhancing rim similar to CT (in panel A), and is without distinct enhancing internal components. 

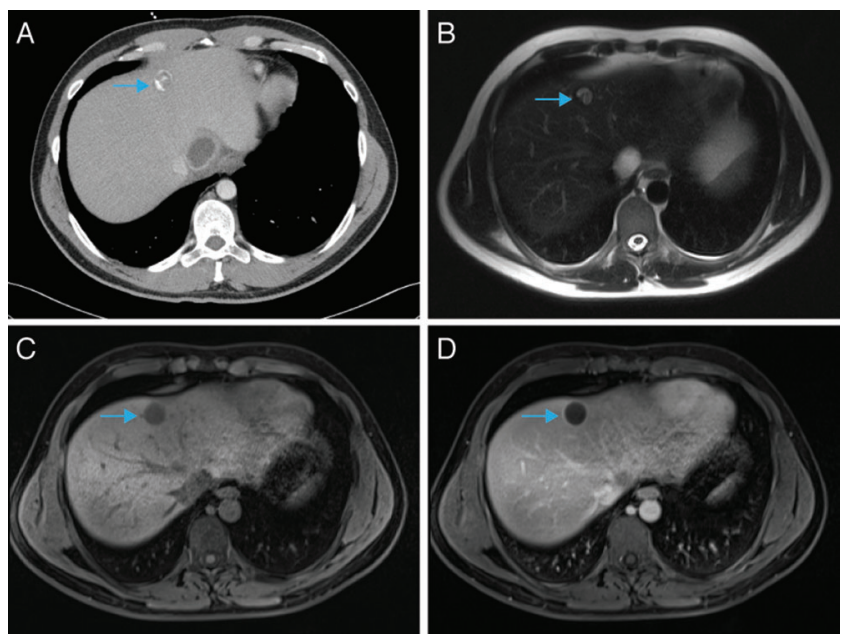

Fig. 2. Superior view of partially calcified hepatic hydatid cyst on CT and MRI (same patient as in Fig. 1). A: Contrast-enhanced CT: hypodense cystic lesion with partially calcified rim and internal components; B: T2-weighted MRI: cystic lesion is mildly hyperintense with low-signal intensity rim and internal components; low-signal intensity is likely due to a combination of calcification and the collagen-rich pericyst; C: Noncontrast T1-weighted MRI with fat saturation: cystic lesion is hypointense; D: Postcontrast T1-weighted MRI with fat saturation: cystic lesion demonstrates an enhancing rim without distinct enhancing internal components. Note: calcification is often inconspicuous on MRI.

(Fig. 3). Daughter vesicles and cysts are rounded structures located peripherally within the dominant cyst, and often demonstrate lower attenuation of the fluid compared with the dominant cyst. ${ }^{2}$

\section{MRI}

While not often needed, MRI may provide additional information not seen on CT. ${ }^{39}$ The signal intensity of CE resembles that of fluid on many MRI sequences. The cyst is hyperintense on T2-weighted images, and is surrounded by a low signal rim, which likely represents a collagen-rich outer layer

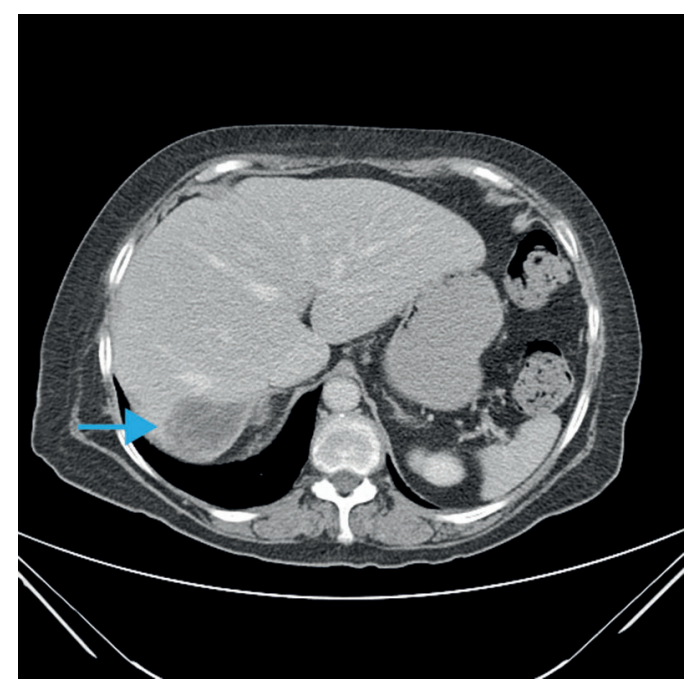

Fig. 3. Contrast-enhanced CT. Complex fluid collection with curvilinear densities (blue arrow), consistent with detachment of the laminated membranes of the endocyst from the pericyst of a hepatic hydatid cyst. (pericyst), and is often described as a characteristic finding of CE (Fig. 1 and 2). ${ }^{2,35}$ Daughter cysts or vesicles, if present, attach to the germinal layer and are hypointense on T1-weighted images relative to the dominant cyst. Collapsed membranes from the pericyst appear as twisted linear structures within the cyst, similar to that seen using CT. Although calcification can be clearly depicted by CT, MRI can detect early irregularities in the wall, thought to represent an impending membrane detachment. 2,38,39

\section{Treatment}

The approach to management and treatment of CE depends on the extent of organ involvement, the number of cysts, presence or absence of cystic-biliary communications, and other factors, such as secondary bacterial infection and hemorrhage. It is, therefore, crucial to assess each individual case to determine the best possible outcome. ${ }^{18}$

\section{Chemotherapy}

Of the chemotherapeutic agents currently available for $C_{\text {, }}$ the two best studied are the benzimidazole carbamates, albendazole and mebendazole. Their primary mechanism of action involves interfering with parasite glucose absorption, resulting in glycogen depletion within the parasitic intracellular organelles. Studies have indicated that the effects of albendazole are superior mebendazole. ${ }^{26,27}$ From drug data reports, it appears that the principle difference between the two is in the breakdown metabolites; the metabolite of albendazole is a potent prodrug that has excellent antihelminthic properties, whereas mebendazole breaks down into multiple, poorly active metabolites. ${ }^{31}$

Medical therapy is indicated in the following cases: 1 ) inoperable cases in primary lung and liver CE with multiple cysts and peritoneal involvement; 2) to reduce cyst pressure, secondary seeding, and risk of recurrence in presurgical and prepuncture cases. Contraindications include: 1 ) large cysts that are likely to rupture; 2 ) inactive or heavily calcified cysts; 3) early pregnancy; 4) chronic hepatic conditions and bone marrow suppressive disorders where treatment results in adverse side effects. ${ }^{19}$ The treatment dosage for a typical 70-kilogram person is $400 \mathrm{mg}$ BID for 28 days. ${ }^{19}$ The most common toxic effect is an elevation of liver enzymes during long-term therapy, which can occur in $20 \%$ of cases. As a drug class, benzimidazoles are also known to have suppressive effects on bone marrow, which usually subside with cessation of the agent. ${ }^{26,27}$ It is, therefore, crucial to monitor hepatic enzymes and complete blood counts. ${ }^{26,27,31}$

The EchinoMEDREV study extrapolated treatment data from various other studies and analyzed the treatment effects of benzimidazoles on patients with liver and peritoneal cysts (staged according to the WHO-IWGE ultrasound classification system). ${ }^{26}$ However, these data only involved treatment of simple cysts $<6 \mathrm{~cm}$ in diameter or highly active cysts, and thus were not sufficient for creating a standard therapy regimen across all cyst sizes, stages, and structures. Nevertheless, data from six centers were gathered to determine relapse rates; highly active CE1 and smaller cysts had the best response, and of those, $25 \%$ reverted to active status after 1.5-2 years of therapy after an initial response. ${ }^{26}$ This can be explained by the incomplete formation of a thick cystic wall in the beginning years of therapy, followed by decreased penetration of therapy due to increased cyst calcification. 
Pakala T. et al: Hepatic echinococcal cysts
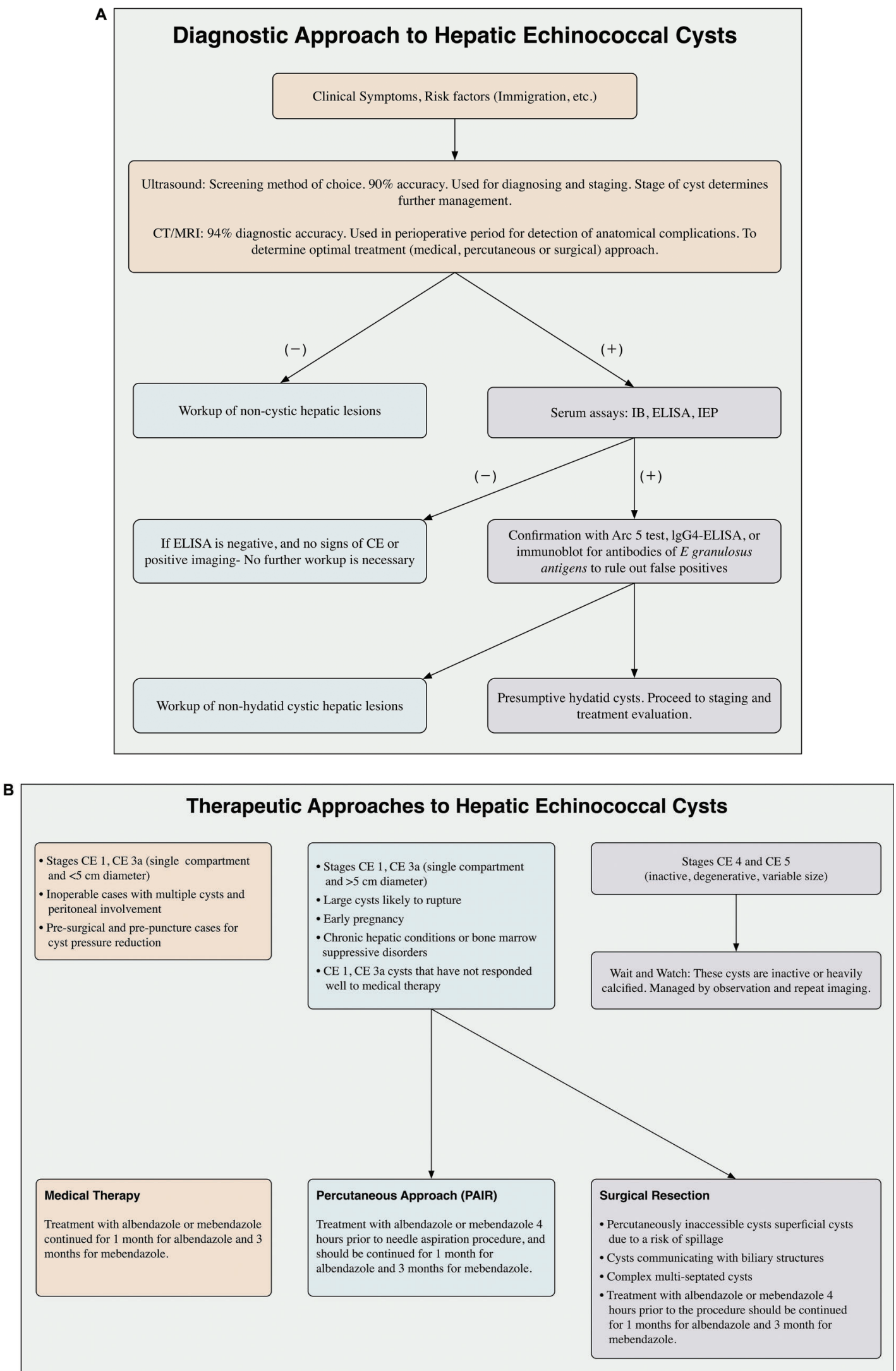

Fig. 4. Management algorithms for cystic echinococcosis. ${ }^{\mathbf{2 4}, \mathbf{2 6}, \mathbf{2 7}, \mathbf{3 6}, \mathbf{3 7}}$ A: Diagnostic approaches; B: Therapeutic approaches. IB, Immunoblot; IEP, Immunoelectrophoresis. 
Another study was a clinical analysis (mostly from one center) of 612 patients with a total of 159 cysts. ${ }^{27}$ Most (50-75\%) of the cysts staged as CE1 (active) were determined to be inactive after initiation of benzimidazole treatment (monitored after 1-2 years), compared to $30-50 \%$ of CE2 and CE3 cysts that were staged as inactive. In addition, $50-60 \%$ of smaller cysts ( $<6 \mathrm{~cm}$ at baseline) responded better to treatment after $1-2$ years compared to $25-50 \%$ of larger cysts.

There is another broad-spectrum, anti-helminthic agent called praziquantel. However, this drug alone is not sufficient as therapy for $\mathrm{CE}$, and is recommended in combination with albendazole, particularly as a preoperative regimen. In a patient cohort with intra-abdominal $C E$, patients that received a preoperative regimen of albendazole plus praziquantel had a greater number of nonviable protoscolices at the time of surgery compared to patients that received albendazole alone. ${ }^{43}$

Variations in inclusion criteria, therapy dose cycles, outcome measures, and follow-up among the included studies on benzimidazole treatment prohibit meta-analysis of these data. It is, therefore, difficult to extrapolate clear-cut treatment regimens based on cyst number or size and posttherapy outcomes.

\section{Watch-and-wait method}

Another treatment strategy is a relatively conservative approach to CE management. In this approach, the hypothesis is that cyst types CE4 and CE5, as shown in the algorithm (Fig. 4), should be left untreated but monitored closely. The fact that some cysts are heavily calcified and remain as fairly inactive structures has been used to justify this strategy. ${ }^{24}$ Follow-up with ultrasound in these cyst types is suggested, as opposed to serologic studies used to assess for activity. Serologic studies are not as reliable given their results vary depending on cyst stage, location, and size.

\section{Surgical management}

Surgical management of echinococcal cysts, most commonly with partial and total cystectomy, has long been considered the definitive cure for $\mathrm{CE} .^{32,34}$ Standard of care even for a surgical approach includes pre- and postprocedure adjunctive drug therapy to prevent secondary seeding of the peritoneal cavity in case of a rupture. According to WHO guidelines, treatment with albendazole or mebendazole should be started four days prior to surgery, and continued after for at least one month with albendazole and for three months with mebendazole. ${ }^{19}$

There are many approaches to the surgical removal, but all must accomplish two goals: cyst removal and obliteration of the cavity. If spillage occurs, immediate washout of the peritoneum should be performed with hypertonic saline and a scoliocidal agent, followed by a longer duration of postprocedure mebendazole therapy, up to six months in some cases. ${ }^{28,31}$ Importantly, a lack of cysto-biliary communications should be confirmed prior to use of hypertonic saline to avoid complications, such as sclerosing cholangitis and pancreatitis. This can be achieved with the use of intraoperative dyes and, if found, careful repair of such communications.

Approaches vary from radical resection to simple cyst resection, but each case varies depending on location, number of cysts, and structural complications, with the ideal approach being whole, simple resection without rupture. ${ }^{32}$
Other approaches range from a more radical pericystectomy, all the way to a conservative approach that includes incision and drainage of cystic fluid, injection of a scoliocidal agent, and aspiration of cyst contents with pericystic tissue removal. An open total pericystectomy uses protoscolicidal agents to sterilize the cyst, followed by removal of the pericystic tissue and contents. In comparison, a closed, total pericystectomy involves removal of the cyst without opening it. A newer surgical approach called subadventitial cystectomy has been developed for liver hydatid disease. ${ }^{45,47}$ The pericyst is a combination of two tissue layers, namely the adventitial layer towards the liver parenchyma and the exocyst layer towards the parasitic cyst. The space between can easily be separated, therefore protecting the layer adjacent to the liver parenchyma and resulting in fewer complications from structural damage and bleeding. The study performed by Chen et $\mathrm{al}^{48}$ showed that subadventitial cystectomy, when compared to pericysectomy (both partial and complete) and hepatic resection, resulted in fewer complications, fewer hospital days, and decreased parasite burden as noted with decreased serum Ig levels when followed one year postoperatively.

In accordance with many recent reports, there has been a progressive increase in surgical approaches because of fewer relapses compared to medical therapy, and also fewer postoperative complications and associated mortality. ${ }^{31}$ In contrast, some studies have shown a higher morbidity and mortality with surgical treatment approaches, along with a relapse rate of $2-25 \% .{ }^{19,33}$ This has shifted the focus of first-line management to less-invasive interventions, thus reserving surgery for complicated cases involving multiple cysts, rupture, bleeding, fistula formation, and compression. However, it is difficult to truly compare surgical and medical management outcomes as there are no prospective clinical trials with long-term follow-up data.

\section{Percutaneous aspiration injection and reaspiration (PAIR)}

This less-invasive approach employs ultrasound or CT-guided aspiration of the cystic fluid. It plays an important role for both confirmation of diagnosis and therapeutic intervention. However, PAIR is not suitable for all cyst types. Prior determination of the number of compartments and the presence of daughter cysts is crucial for successful treatment with this strategy. ${ }^{36}$ Indications for PAIR include WHO-IWGE classification CE1 and CE3a cysts (single compartment cysts) $<5 \mathrm{~cm}$ that have not responded well to medical therapy, and in combination with medical therapy for cysts $>5 \mathrm{~cm} .{ }^{34,38}$ Contraindications for PAIR include percutaneously inaccessible cysts, superficial cysts due to a risk of spillage, cysts communicating with biliary structures, inactive cysts, and complex multiseptated cysts. ${ }^{39}$

The procedure involves aspiration, injection of scolicidal agent, and the reaspiration of contents. The fluid that is initially aspirated is evaluated for viable proctoscolices, which confirms the diagnosis. It is also evaluated for biliary-cystic communication by testing for the presence of bilirubin in the fluid, which can also be determined prior to PAIR using cholangiography or endoscopic retrograde cholangiopancreatography. ${ }^{2}$ The scolicidal agent that is injected is left for approximately 15 minutes, after which there is separation of the germinal membrane from the surrounding cyst. Currently, three solutions are most commonly used: $70-95 \%$ ethanol, $15-20 \%$ hypertonic saline, or cetrimide solution. ${ }^{19}$ 
The procedure also involves close monitoring for complications of anaphylaxis. Treatment with albendazole or mebendazole four hours prior to the procedure should be continued for one month postoperatively for albendazole, and for three months with mebendazole. This pre- and post-treatment reduces the risk for recurrence and secondary intraperitoneal seeding. ${ }^{19,34}$

Post-PAIR, serial sonographic imaging is performed to monitor the patient's response. A good response is determined by the presence of one or more of the following factors: reduction in the size of the cavity, increased wall calcification, increased areas of solidification in the cyst, and increased echogenicity of the cyst (consistent with a pseudomass appearance). ${ }^{35,37}$ In a meta-analysis study that compared 769 patients undergoing PAIR plus mebendazole or albendazole to 952 patients that underwent surgery, better clinical and parasitologic cure was observed with PAIR plus chemotherapy, along with lower rates of morbidity, significantly fewer hospital days ( $2.4 \mathrm{~d} v s 15 \mathrm{~d}$ ), and lower disease recurrence rates. ${ }^{41}$ In addition, complications of anaphylaxis, cyst infection, biliary fistula, and intra-abdominal abscess occurred more frequently in the surgical population.

\section{Management of CE based on WHO-IWGE staging}

As shown in Figure 4, current standards for monotherapy are for WHO-IWGE stages CE1 and CE3a (cysts with single compartment and $<5 \mathrm{~cm}$ in diameter). ${ }^{19}$ Treatment is aimed for continuous therapy from 1-3 months up to six months, depending on the clinical scenario. In multicystic liver with cysts $<5 \mathrm{~cm}$, peritoneal cysts, or areas where percutaneous approach is not feasible, monotherapy is also justified. PAIR, as mentioned above, is effective in smaller WHO-IWGE classification CE1 and CE3a cysts that have not responded well to medical therapy or in combination with medical therapy for larger cysts. ${ }^{35,38}$ Multicompartment cyst types or cysts that contain daughter cysts (types CE2 and CE3b) require surgery in combination with medical therapy or a different type of percutaneous intervention (non-PAIR) due to the high risk for relapse after PAIR in these patients. The alternative percutaneous intervention is generally performed with a largebore catheter that is able to evacuate the entire cyst as opposed to obliterating the germinal layer with a scolicidal agent. ${ }^{48}$ Cyst types CE4 and CE5 are inactive cysts and are managed by observation. ${ }^{24,35,37}$

\section{Follow-up period}

Follow-up is recommended initially every six months for the first two years, and then once a year depending on the appropriate clinical setting. ${ }^{39}$ As mentioned earlier, patients undergoing chemical therapy require serial liver function tests and leukocyte counts to monitor for adverse reactions. In CE, it is difficult to assess the frequency of relapses. Therefore, monitoring with ultrasound is sometimes performed for up to ten years, a duration for which recurrences have been reported despite treatment. ${ }^{39}$ In the post-treatment phase, serologic studies, often with Ig levels, are difficult to interpret because they may indicate residual disease as opposed to a disease recurrence. In many cases, they remain elevated despite appropriate therapy or complete resection, which is why they are often used in combination with imaging studies during follow-up to detect cystic activity. ${ }^{19}$

\section{Conclusion}

Hepatic echinococcal cysts, although fairly uncommon in North America, should be considered in the differential diagnosis of hepatic cysts, particularly in patients with exposure risk, such as those who have traveled to or emigrated from a region of high prevalence. Serum antibody assays generally have low sensitivities, but antigen assays may be of value. Imaging is crucial in determining cyst stage, size, and location and complications. It can also be helpful in assessing the suitability of a minimally invasive PAIR approach. Uncomplicated active cysts can be managed with chemotherapy alone or in combination with a PAIR approach. Uncomplicated, inactive cysts can be managed with the "watch-and-wait" strategy. Complicated cysts with structural involvements of the biliary system require surgery.

\section{Conflict of interest}

None

\section{Author contributions}

Conceiving the idea and edtiting the manuscript (GW), writing the manuscript (TP), providing the image and legends (MM).

\section{References}

[1] Siracusano A, Teggi A, Ortona E. Human Cystic Echinococcosis: Old Problems and New Perspectives. Interdiscip Perspect Infect Dis 2009:474368. doi: 10. $1155 / 2009 / 474368$.

[2] Pedrosa I, Saíz A, Arrazola J, Ferreirós J, Pedrosa CS. Hydatid disease: Radiologic and pathologic features and complications. Radiographics 2000; 20: 795-817. doi: 10.1148/radiographics.20.3.g00ma06795.

[3] Lewall DB: Hydatid disease: Biology, pathology, imaging and classification. Clin Radiol 1998;52:863-874. doi: 10.1016/S0009-9260(98)80212-2.

[4] Gottstein B. Hydatid Disease, Major Tropical syndromes by body system. Systemic infections. Chap 2000;169, section 6.

[5] Siracusano A, Delunardo F, Teggi A, Ortona E. Cystic echinococcosis: aspects of immune response, immunopathogenesis and immune evasion from the human host. Endocr Metab Immune Disord Drug Targets 2012;12:16-23. doi: $10.2174 / 187153012799279117$.

[6] Zhang W, Ross A, and McManus D. Mechanisms of Immunity in Hydatid Disease: Implications for Vaccine Development. J Immunol 2000;181: 6679-6685. doi: 10.4049/jimmunol.181.10.6679.

[7] Zhang W, Wen H, Li J, Lin R, McManus D. Immunology and Immunodiagnosis of Cystic Echinococcosis: An Update. Clin Dev Immunol 2012; 2012:101895. doi: $10.1155 / 2012 / 101895$

[8] Zhang W, Li J, McManus D. Concepts in Immunology and Diagnosis of Hydatid Disease. Clin Microbiol Rev 2003;16:18-36. doi: 10.1128/CMR.16.1.18-36. 2003.

[9] García MB, Lledías JP, Pérez IG, Tirado VV, Pardo LF, Bellvís LM, et al. Primary Super-Infection of Hydatid Cyst-Clinical Setting and Microbiology in 37 Cases. Am J Trop Med Hyg 2010;82:376-378. doi: 10.4269/ajtmh.2010. 09-0375.

[10] Siracusano A, Delunardo F, Teggi A, Ortona E. Host-Parasite Relationship in Cystic Echinococcosis: An Evolving Story. Clin Dev Immunol 2012;2012: 639362. doi: 10.1155/2012/639362.

[11] Scherer K, Gupta N, Caine W, Panda M. Differential Diagnosis and Management of a Recurrent Hepatic Cyst: A Case Report and Review of Literature. J Gen Intern Med 2009;24:1161-1165. doi: 10.1007/s11606-009-1062-1.

[12] Gottstein B. An immunoassay for the detection of circulating antigens in human echinococcosis. Am J Trop Med Hyg 1984;33:1185-1191.

[13] Ravinder P, Parija S, Rao K. Evaluation of human hydatid disease before and after surgery and chemotherapy by demonstration of hydatid antigens and antibodies in serum. J Med Microbiol 1997;46:859-864. doi: 10.1099/ 00222615-46-10-859.

[14] Sadjjadi S, Sedaghat F, Hosseini S, Sarkari B. Serum Antigen and Antibody Detection in Echinococcosis: Application in Serodiagnosis of Human Hydatidosis. Korean J Parasitol 2009;47:153-157. doi: 10.3347/kjp.2009.47. 2.153 . 
[15] Liance $M$, Janin V, Bresson-Hadni S, Vuitton D-A, Houin R, Piarroux R. Immunodiagnosis of Echinococcus Infections: Confirmatory Testing and Species Differentiation by a New Commercial Western Blot. J Clin Microbiol 2000; 38:3718-3721.

[16] Ortona E, Riganò R, Margutti P, Notargiacomo S, Ioppolo S, Vaccari S, et al, Native and recombinant antigens in the immunodiagnosis of human cystic echinococcosis. Parasite Immunol 2000;22:553-559. doi: 10.1046/j.13653024.2000.00336.x

[17] Langer J, Rose D, Keystone J, Taylor B, Langer B. Diagnosis and management of hydatid disease of the liver. A 15 -year North American experience. Ann Surg 1984;199:412-417. doi: 10.1097/00000658-198404000-00007.

[18] Symeonidis N, Pavlidis T, Baltatzis M, Ballas K, Psarras K, Marakis G, et al. Complicated liver echinococcosis: 30 years of experience from an endemic area. Scand J Surg 2013;102:171-177. doi: 10.1177/1457496913491877.

[19] Brunetti E, Kern P, Vuitton DA; Writing Panel for the WHO-IWGE. Expert consensus for the diagnosis and treatment of cystic and alveolar echinococcosis in humans. Acta Trop 2010;114:1-16. doi: 10.1016/j.actatropica. 2009.11.001.

[20] Macpherson C, Milner R. Performance characteristics and quality control of community based ultrasound surveys for cystic and alveolar echinococcosis. Acta Trop 2003;85:203-209. doi: 10.1016/S0001-706X(02)00224-3.

[21] WHO Informal Working Group. International classification of ultrasound images in cystic echinococcosis for application in clinical and field epidemiological settings. 2003;85:253-261.

[22] Marrone G, Crino' F, Caruso S, Mamone G, Carollo V, Milazzo M, et al. Multidisciplinary imaging of liver hydatidosis. World J Gastroenterol 2012;18: 1438-1447. doi: 10.3748/wjg.v18.i13.1438.

[23] Stojkovic M, Rosenberger K, Kauczor H-U, Junghanss T, Hosch W. Diagnosing and Staging of Cystic Echinococcosis: How Do CT and MRI Perform in Comparison to Ultrasound? PLoS Negl Trop Dis 2012;6:e1880.

[24] Piccoli L, Tamarozzi F, Cattaneo F, Mariconti M, Filice C, Bruno A, et al. Longterm Sonographic and Serological Follow-up of Inactive Echinococcal Cysts of the Liver: Hints for a "Watch-and-Wait" Approach. PLoS Negl Trop Dis 2014; 8:e3057. doi: 10.1371/journal.pntd.0003057.

[25] Yagci G, Ustunsoz B, Kaymakcioglu N, Bozlar U, Gorgulu S, Simsek A, et al. Results of surgical, laparoscopic, and percutaneous treatment for hydatid disease of the liver: 10 years' experience with 355 patients. World J Surg 2005;29:1670-1679. doi: 10.1007/s00268-005-0058-1.

[26] Stojkovic M, Zwahlen M, Teggi A, Vutova K, Cretu CM, Virdone R, etal. Treatment Response of Cystic Echinococcosis to Benzimidazoles: A Systematic Review. PLoS Negl Trop Dis 2009;3:e524. doi: 10.1371/journal.pntd.0000524.

[27] Teggi A, Lastilla MG, De Rosa F. Therapy of human hydatid disease with mebendazole and albendazole. Antimicrob Agents Chemother 1993;37: 1679-1684. doi: 10.1128/AAC.37.8.1679.

[28] Paksoy Y, Odev K, Sahin M, Arslan A, Koç O. Percutaneous treatment of liver hydatid cysts: comparison of direct injection of albendazole and hypertonic saline solution. Am J Roentgenol 2005;185:727-734. doi: 10.2214/ajr.185. 3.01850727.

[29] Dawson J, Stamatakis J, Stringer M, Williams R. Surgical retreatment of hepatic hydatid disease. $\mathrm{Br}$ J Surg 1988;75:946-950. doi: 10.1002/bjs. 1800751004.

[30] Sözen S, Emir S, Tükenmez M, Topuz Ö. The results of surgical treatment for hepatic hydatid disease. Hippokratia 2011;15:327-329.

[31] Smego R, Sebanego P. Treatment options for hepatic cystic echinococcosis. Int J Infect Dis 2005;9:69-76. doi: 10.1016/j.ijid.2004.08.001.

[32] Tuxun T, Zhang JH, Zhao JM, Tai QW, Abudurexti M, Ma HZ, et al. World review of laparoscopic treatment of liver cystic echinococcosis-914 patients. Int J Infect Dis 2014;24:43-50. doi: 10.1016/j.ijid.2014.01.012.
[33] McNanus D, Gray D, Zhang W, Yang Y. Diagnosis, treatment, and management of Echinococcosis. BMJ 2012;344:e3866. doi: 10.1136/bmj.e3866.

[34] Junghanss T, da Silva AM, Horton J, Chiodini PL, Brunetti E. Clinical management of cystic echinococcosis: state of the art, problems, and perspectives. Am J Trop Med Hyg 2008;79:301.

[35] Rajesh R, Dalip D, Anupam J, Jaisiram A. Effectiveness of PunctureAspiration-Injection-Reaspiration in the Treatment of Hepatic Hydatid Cysts. Iran J Radiol 2013; 10:68-73. doi: 10.5812/iranjradiol.7370.

[36] Golemanov B, Grigorov N, Mitova R, Genov J, Vuchev D, Tamarozzi F, et al. Efficacy and Safety of PAIR for Cystic Echinococcosis: Experience on a Large Series of Patients from Bulgaria. Am J Trop Med Hyg 2011;84:48-51. doi: 10. 4269/ajtmh.2011.10-0312.

[37] Rinaldi F, Brunetti E, Neumayr A, Maestri M, Goblirsch S, Tamarozzi F. Cystic echinococcosis of the liver: A primer for hepatologists. World J Hepatol 2014; 6:293-305. doi: 10.4254/wjh.v6.i5.293.

[38] Beggs I. The radiology of hydatid disease. Am J Roentgenol 1985;145:639648. doi: 10.2214/ajr.145.3.639.

[39] Marani SA, Canossi GC, Nicoli FA, Alberti GP, Monni SG, Casolo PM. Hydatid disease: MR imaging study. Radiology 1990;175:701-706. doi: 10.1148/ radiology.175.3.2343117.

[40] Hunter G, Strickland G. Hunter's Tropical medicine and emerging infectious diseases. 8th ed. Philadelphia: Saunders, 2000:866-876.

[41] Smego RA Jr, Bhatti S, Khaliq AA, Beg MA. Percutaneous AspirationInjection-Reaspiration Drainage plus Albendazole or Mebendazole for Hepatic Cystic Echinococcosis: A Meta-analysis. Clin Infect Dis 2003;37: 1073-1083. doi: 10.1086/378275.

[42] Lissandrin R, Tamarozzi F, Piccoli L, Tinelli C, Silvestri A, Mariconti M, et al. Factors Influencing the Serological Response in Hepatic Echinococcus granulosus Infection. Am J Trop Med Hyg 2016; 94:166-171. doi: 10. 4269/ajtmh.15-0219.

[43] Cobo F, Yarnoz C, Sesma B, Fraile P, Aizcorbe M, Trujillo R, et al. Albendazole plus praziquantel versus albendazole alone as a pre-operative treatment in intra-abdominal hydatisosis caused by Echinococcus granulosus. Trop Med Int Health 1998;3: 462-466. doi: 10.1046/j.1365-3156.1998.00257.x.

[44] Nunnari G, Pinzone MR, Gruttadauria S, Celesia BM, Madeddu G, Malaguarnera $\mathrm{G}$, et al. Hepatic echinococcosis: Clinical and therapeutic aspects. World J Gastroenterol 2012;18:1448-1458. doi: 10.3748/wjg.v18.i13.1448.

[45] Lv H, Jiang Y, Peng X, Zhang S, Wu X, Yang H, et al. Total subadventitial cystectomy for the treatment of 30 patients with hepatic hydatid cysts. Chin J Gen Surg 2002;17:529-530.

[46] Otero-Abad B, Torgerson PR. A Systematic Review of the Epidemiology of Echinococcosis in Domestic and Wild Animals. Garcia. PLoS Negl Trop Dis 2013; 7:e2249. doi: 10.1371/journal.pntd.0002249.

[47] Peng X, Li J, Wu X, Zhang S, Niu J, Chen X, et al. Detection of Osteopontin in the pericyst of human hepatic Echinococcus granulosus. Acta Trop 2006; 100:163-171. doi: 10.1016/j.actatropica.2006.08.013.

[48] Chen $X$, Chen $X$, Shao $Y$, Zhao J, Li H, Wen $H$. Clinical Outcome and Immune Follow-Up of Different Surgical Approaches for Human Cyst Hydatid Disease in Liver. Am J Trop Med Hyg 2014;91: 801-805. doi: 10.4269/ajtmh. 14-0177.

[49] Craig PS. Immunodiagnosis of Echinococcus granulosus. In: Andersen FL, ed. Compendium on CE with special references to the Xinjiang Uygur Autonomous Region, the People's Republic of China. Provo: Brigham Young University, 1993:85-118.

[50] Pawlowski ZS. Critical points in the clinical management of cystic echinococcosis: a revised review. In: Andersen FL, Ouhelli H, Kachani M, eds. Compendium on cystic echinococcosis in Africa and in Middle East Countries with special reference to Morocco. Provo: Brigham Young University Print Services, 1997:119-135. 